\title{
Variaciones termométricas en la planta del pie y piernas valorada en corredores antes y después de correr $30 \mathrm{~km}$
}

\author{
Thermometric Variations on the Foot Sole and Legs of Runners before and \\ after Running $30 \mathrm{~km}$
}

\author{
Vanessa Robles Dorado \\ Podóloga. Clínica de Podología.
}

Correspondencia:

Vanessa Robles Dorado

Avd. República Argentina $n^{\circ} 311^{\circ} \mathrm{izq}$

E- 24004 León

Correo-e: vanessaroblesdorado@yahoo.es

La autora declara no tener ningún tipo de interés económico o comercial.

Fecha de recepción: 10 de marzo de 2014

Fecha de aceptación: 19 de enero de 2015

\begin{abstract}
RESUMEN
La termometría es una técnica no invasiva que permite cuantificar los cambios en la temperatura cutánea y evaluarla de forma cuantitativa. El aumento significativo de la temperatura puede indicar la existencia de patología. Se ha demostrado que la actividad muscular induce procesos de transferencia de calor entre los músculos y las capas superficiales de tejido. En este estudio queremos cuantificar los cambios de temperatura que se producen en los músculos del pie y miembro inferior tras una carrera de $30 \mathrm{~km}$, para ello hemos utilizado una cámara termográfica de alta resolución. Contamos con la colaboración voluntaria de 32 sujetos sanos a los que procedimos a tomar fotografías de la planta del pie, parte anterior de la pierna, parte posterior de la pierna, parte anterior del muslo y parte posterior del muslo en dos etapas, primero antes de la carrera y segunda toma después de la carrera de $30 \mathrm{~km}$, de esta manera pudimos valorar si había o no variación de temperatura en las zonas seleccionadas.

Tras el análisis de los datos obtenidos encontramos significativas variaciones térmicas en Talón, cabeza primer metatarsiano, cabeza segundo metatarsiano, cabeza tercer metatarsiano, cabeza cuarto metatarsiano, cabeza quinto metatarsiano, apófisis estiloides quinto metatarsiano, arco longitudinal interno, maléolo interno, maléolo externo, peroneo lateral largo, vasto interno, vasto externo, recto femoral, tensor de la fascia lata, inserción cuádriceps, gemelo interno, tendón de Aquiles y Biceps femoral.
\end{abstract}

Palabras clave: termografía infrarroja; carrera; termometría.

\begin{abstract}
Thermometry is a non-invasive technique to measure changes on the cutaneous temperature and assess it in a quantitative way. The significant raising of the temperature may indicate the existence of pathology. It has been shown that muscular activity inducts heat transference processes between muscles and the superficial layers of tissue. In this study we want to quantify the temperature changes in the foot muscles and in the lower limb after a $30 \mathrm{~km}$ race. In order to do so, we have used a high resolution thermographic camera. We count on the voluntary collaboration of 32 healthy people, and we proceeded to take pictures of their foot sole, the front and back of their legs, the front and back parts of their thigh in two stages; The first one before the race and the second after the $30 \mathrm{~km}$ race, so we could assess whether there was temperature variation in the selected areas.

After de analysis of the obtained data we found significant thermic variations in the heel, first metatarsal head, second metatarsal head, third metatarsal head, fourth metatarsal head, fifth metatarsal head, styloid process fifth metatarsal, inner longitudinal arch, inner malleolus, external malleolus, peroneus longus, vastus medialis, vastus lateralis, rectus femoris, tensor fasciae latae, quadriceps insertion, medial gastrocnemius, Achilles tendon and biceps femoris.
\end{abstract}

Key words: infrared thermography; race; thermometry.

Referencia normalizada: Robles Dorado, V. Variaciones termométricas en la planta del pie y piernas valorada en corredores antes y después de correr 30 km. Rev. Int. Cienc. Podol. 2016; 10(1): 31-40.

Sumario: 1. Introducción. 2. Materia y métodos. 3. Resultados. 4. Discusión. 5. Conclusión. Bibliografía. 


\section{INTRODUCCIÓN}

El Diccionario terminológico de Ciencias Médicas explica que la termometría en medicina se encarga de la medición de las temperaturas del cuerpo y del estudio de sus variaciones durante las enfermedades ${ }^{1}$. La temperatura del cuerpo ha sido desde hace muchos años una parte importante de la medicina ${ }^{2}$.

La termografía aplicada en la medicina, es una técnica no invasiva, que permite al podólogo cuantificar los cambios en la temperatura superficial de la piel y evaluarla de forma cuantitativa $^{3}$. Existe una gran relación entre la variación de temperatura y la posibilidad de sufrir patología ${ }^{4}$. Este método ha ido avanzando considerablemente desde que Lawson, R en 1957 descubrió que los pacientes con cáncer tienen una mayor temperatura en la zona ${ }^{5}$.

Un dispositivo de exploración infrarroja se utiliza para convertir la radiación infrarroja emitida desde la superficie de la piel en una imagen termográfica o termograma. El espectro de colores indica el aumento o disminución de la cantidad de radiación infrarroja que se emite desde la superficie del cuerpo.

El mayor valor clínico de la termografía es su alta sensibilidad a la patología en los sistemas vasculares, musculares, nervioso y óseo, y como tal puede contribuir al diagnóstico efectuado por el médico. El aumento significativo de temperatura puede indicar la existencia de patología ${ }^{4,5}$. Esta técnica se ha utilizado en estudios anteriores para determinar la posible afectación en el pie diabético ${ }^{6,7}$.

Se ha demostrado que la actividad muscular induce procesos de transferencia de calor entre los músculos y las capas superficiales del tejido, lo que a su vez se traduce en variaciones de la temperatura cutánea ${ }^{3,8}$.

El mantenimiento de la postura ortostática se obtiene a través de la compleja actividad antigravedad de los músculos, se espera que con la variación de la postura también se observen variaciones de la temperatura que serán percibidas en las imágenes de infrarrojos de las cámaras de alta resolución térmica. En estudios anteriores utilizaron cámaras termométricas para observar las variaciones térmicas de la piel en oficinistas para valorar la posibilidad de sufrir lesiones en el miembro superior ${ }^{9}$, para valorar las variaciones térmicas durante el ejercicio en personas con entrenamiento y personas sin ningún tipo de entrenamiento, y comprobaron que las personas con entrenamiento tienen un mayor control de la temperatura ${ }^{10}, \mathrm{y}$ para evaluar la posibilidad de padecer lesiones en la rodilla en deportistas ${ }^{11}$.

Por lo tanto, la alta resolución de la imagen de infrarrojos proporcionan una evaluación cuantitativa de los efectos térmicos cutáneos posiblemente asociados con el trabajo muscular durante la carrera ${ }^{12}$.

El objetivo general de este estudio fue cuantificar los cambios de temperatura que se producen en los músculos del pie y miembro inferior tras una carrera de 30 kilometros de distancia.

Los objetivos específicos han sido medir los cambios de temperatura que se producen en los puntos de apoyo del pie, así como medir los cambios de temperatura que se producen en los músculos del miembro inferior tras una carrera de 30 kilometros de distancia.

El objetivo de este estudio fue probar la capacidad de IR de imagen para describir y medir cuantitativamente la temperatura cutánea adaptada a corto plazo y potencialmente asociada a los cambios de postura durante la carrera.

\section{MATERIAL Y MÉTODOS}

\subsection{Los sujetos}

Treinta y dos voluntarios sanos, sin ninguna patología, 28 varones y 4 mujeres se inscriben en este estudio. Las características demográficas de los participantes se muestran en la Tabla 1. En todos los sujetos se ha utilizado el MMII derecho y el MMII izquierdo. Todos los corredores llevan el mismo calcetín pero calzado diferente.

Con una cámara termográfica de infrarrojos se procedió a tomar fotografías de la planta del pie, parte anterior de la pierna, parte anterior del muslo, parte posterior de la pierna y parte posterior del muslo antes de correr y después de correr $30 \mathrm{~km}$.

Con estas fotografías, utilizando el programa informático FlirQuickReport podemos valorar de forma cuantitativa las temperaturas de distintos grupos musculares y estructuras óseas 


\begin{tabular}{rcc} 
Edad & $\mathbf{X} \pm$ SD & Rango \\
\hline Años que lleva corriendo & $8,78 \pm 6,37$ & $32,41-45,15$ \\
\hline Tiempo que lleva praticando deporte & $8,22 \pm 4,37$ & $3,13-13,87$ \\
Número de deportes que practica & $42,56 \pm 2,34$ & $3,31-13,87$ \\
Talla & $174,78 \pm 6,74$ & $40,22-44,9$ \\
Peso & $70,00 \pm 10,36$ & $168,04-181,52$ \\
\hline Volumen de masa corporal & $22,81 \pm 2,21$ & $20,64-80,36$ \\
\hline
\end{tabular}

Tabla 1. Características demográficas de los sujetos.
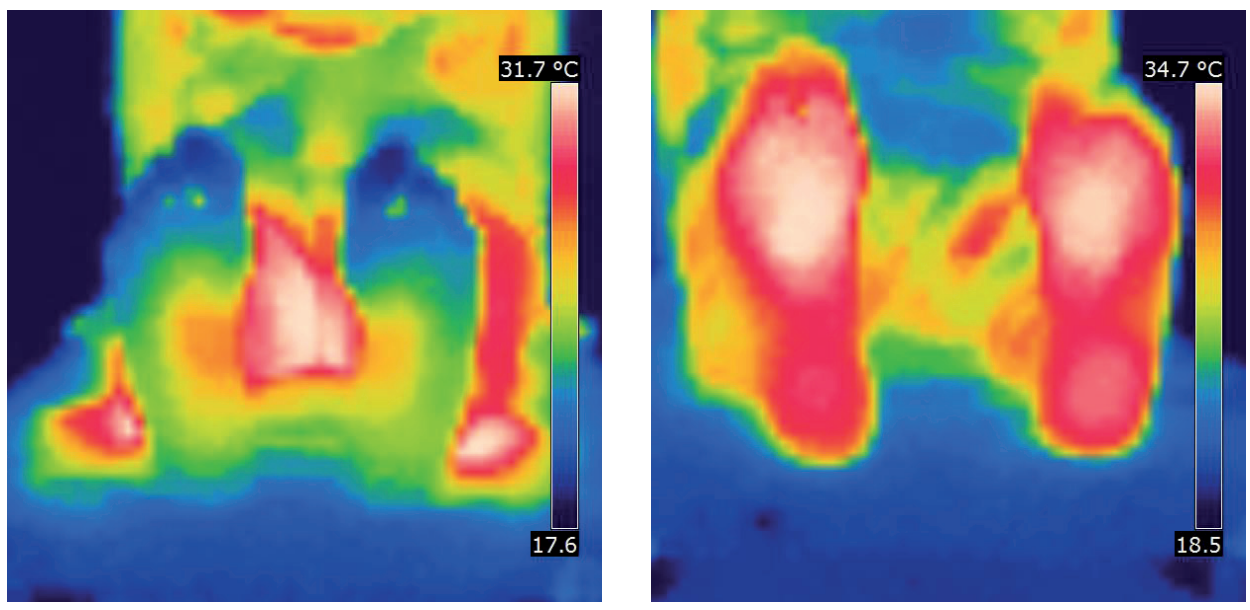

Imagen 1. A zona plantar antes de correr y B zona plantar después de correr.

y así determinar qué zonas han sufrido una mayor sobrecarga durante la carrera.

De los treinta y dos voluntarios sólo han podido incluirse treinta en el estudio, debido a que las imágenes tomadas a dos de los sujetos no tienen suficiente calidad.

Todos los voluntarios firmaron una autorización para ser incluidos en el estudio.

\subsection{Imagen de Infrarrojos}

Antes de comenzar las mediciones todos los sujetos rellenan un cuestionario previo en el que detallan datos de identificación personal, edad, talla y peso. Todos van a correr con su calzado deportivo habitual (sin utilizar planti- llas ortopédicas ni modificaciones del calzado). Se les explica cómo se va a proceder a la toma de las fotografías y que estas se van a realizar en dos momentos, antes de correr y después de correr $30 \mathrm{~km}$.

La primera imagen se toma en decúbito supino, sobre una camilla, y se captura la planta de ambos pies, aquí vamos a valorar las variaciones termométricas de talón, cabeza primer metatarsiano, cabeza segundo metatarsiano, cabeza tercer metatarsiano, cabeza cuarto metatarsiano, cabeza quinto metatarsiano, apófisis estiloides quinto metatarsiano y arco longitudinal interno. (Imagen 1. A antes de correr y B después de correr) 
La segunda imagen se tomará con los sujetos de pie, erguidos y tomaremos una fotografía de la parte anterior de la pierna, donde valoraremos maléolo interno, maléolo externo y los vientres musculares de peroneo lateral largo y tibial anterior. (Imagen 2. A antes de correr y B después de correr)

La tercera imagen en la misma posición que la anterior pero captaremos la parte anterior del muslo, para posteriormente valorar los vientres musculares de vasto interno, vasto externo, recto femoral, tensor de la fascia lata y la inserción del cuádriceps. (Imagen 3. A antes de correr y B después de correr)

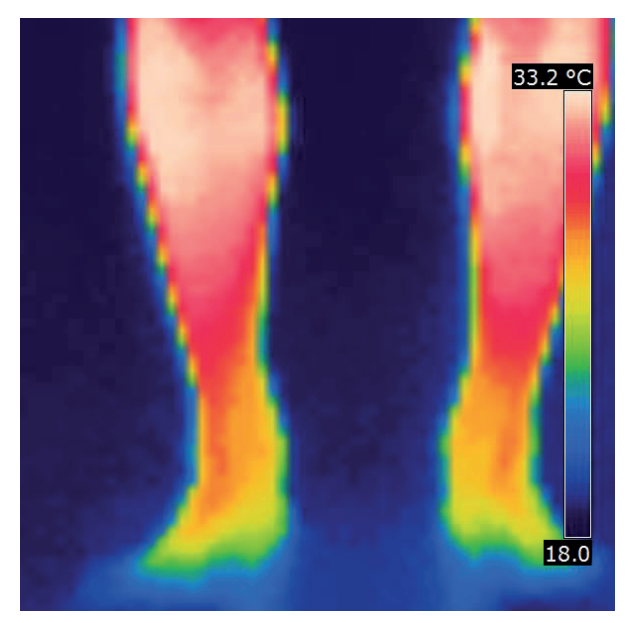

La cuarta imagen también con los sujetos en pie y erguidos será de la parte posterior de la pierna donde podremos valorar las variaciones termométricas de los vientres musculares de gemelo interno, gemelo externo y de la inserción del tendón de Aquiles. (Imagen. 4 A antes de correr y B después de correr)

La quinta y última imagen en la misma posición que en la anterior se capturará una imagen de la parte posterior del muslo, en esta zona se apreciarán los vientres musculares de los músculos semimembranoso, semitendinoso y bíceps femoral. (Imagen 5. A antes de correr y $\mathrm{B}$ después de correr)

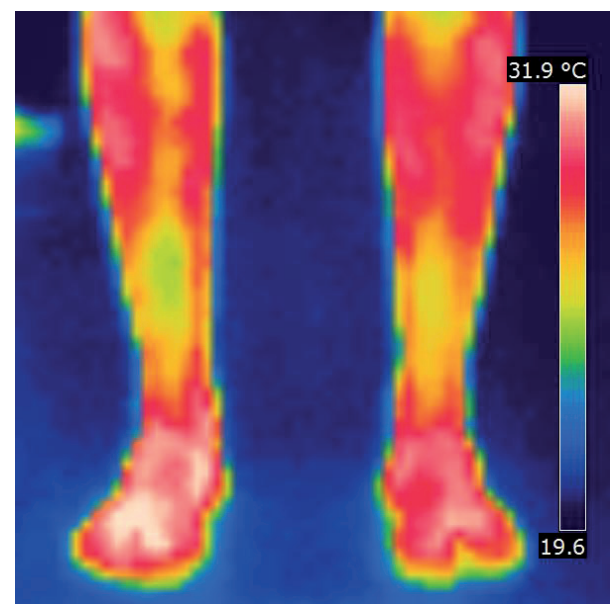

Imagen 2. A Parte anterior pierna antes de correr y B Parte anterior pierna después de correr.
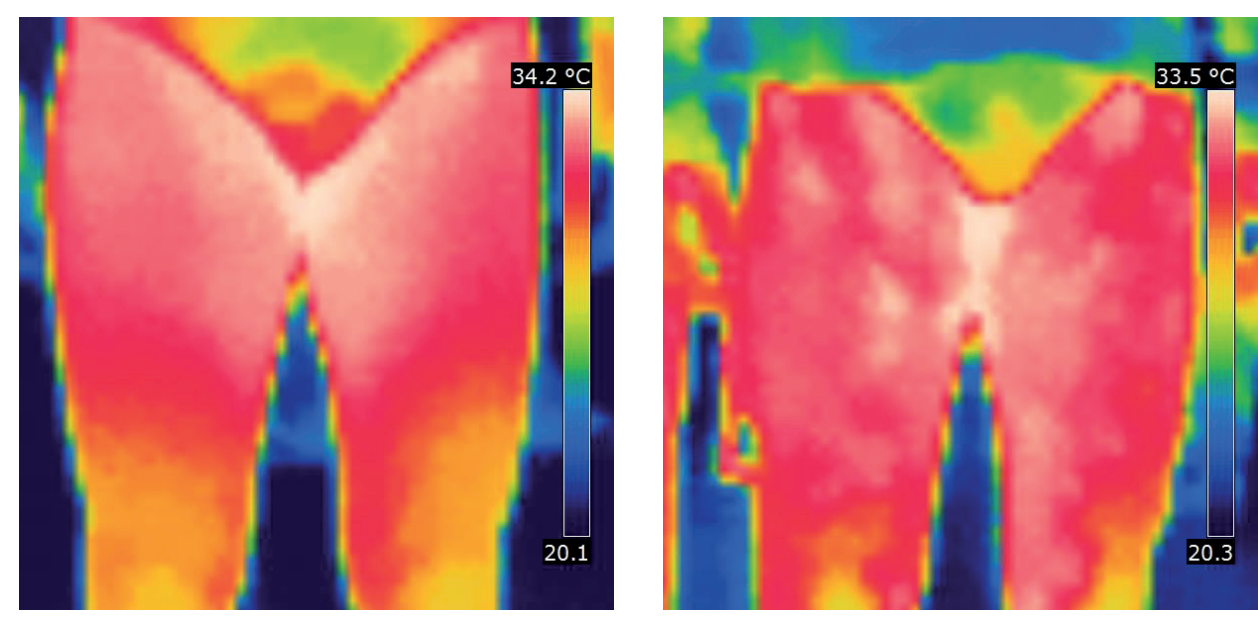

Imagen 3. A Parte anterior muslo antes de correr y B Parte anterior muslo antes de correr. 
Las imágenes de infrarrojos consisten en la grabación de termografías de alta resolución de diferentes segmentos del cuerpo, en puntos de vista anterior y posterior.

Cinco instantáneas de cada uno de los corredores fueron tomadas antes de correr y otras cinco instantáneas después de correr $30 \mathrm{~km}$, por lo que en total se tomaron 10 instantáneas de cada corredor. Las imágenes se grabaron en un archivo de imagen para su posterior análisis.

\subsection{Análisis de datos}

Para la captura de las imágenes se utilizó una cámara térmica con una alta resolución y equi- pamiento elevado que incluye software, el núcleo de la cámara es un microbolómetro no refrigerado ( Uncooled Focal Plane Array) con una resolución de 160 x 120 píxeles. La cámara térmica se ha diseñando de forma ergonómica para el manejo con una sola mano. Gracias a su peso ínfimo de sólo $750 \mathrm{~g}$ es ideal para el análisis de máquinas e instalaciones, para la termografía de construcción y tantas otras aplicaciones. La cámara térmica PCE-TC 3 (PCE Ibérica S.L, Tobarra, España) le ofrece una exactitud de medición de máximo $\pm 2{ }^{\circ} \mathrm{C}$ o $\pm 2 \%$ en un rango de temperatura de $-10{ }^{\circ} \mathrm{C}$ hasta +250 ${ }^{\circ} \mathrm{C}$, con una sensibilidad de sólo $0,15^{\circ} \mathrm{C}$. En la
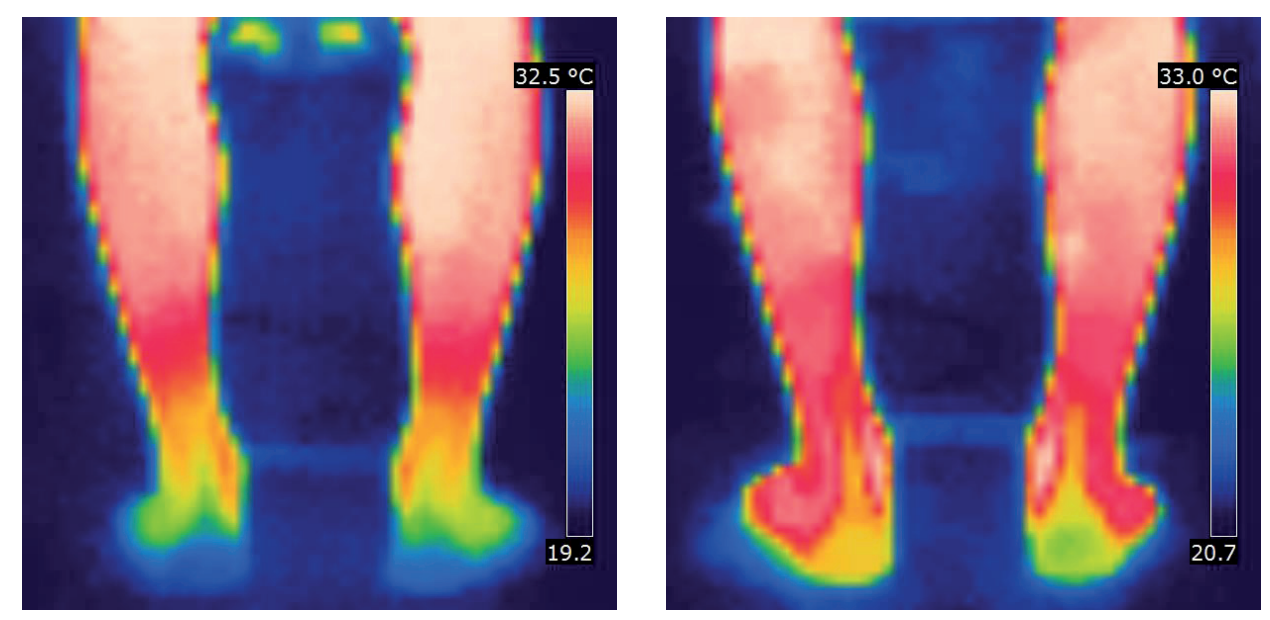

Imagen 4. A Parte posterior pierna antes de correr y B Parte posterior pierna después de correr.
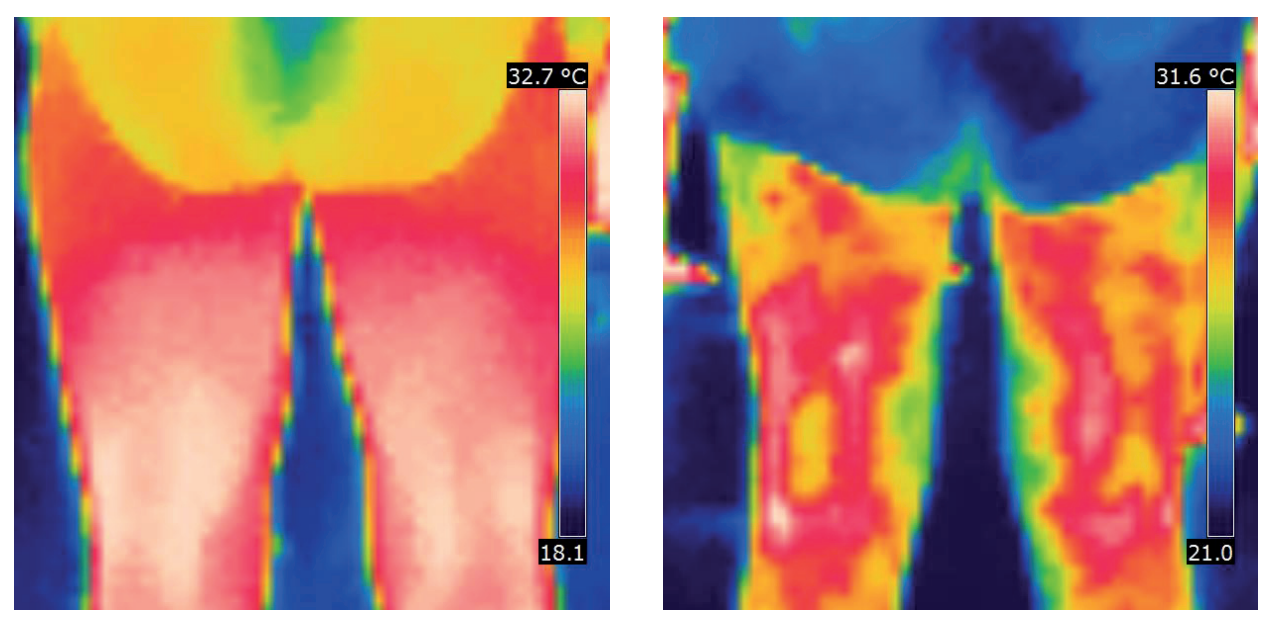

Imagen 5. A Parte posterior muslo antes de correr y B Parte posterior muslo después de correr. 
pantalla de color de la cámara puede desplazar in situ los dos cursores en los diferentes puntos y leer directamente la temperatura correspondiente. También tiene la posibilidad con esta cámara térmica de encontrar y ver de forma automática el punto de medición más caliente y el más frío, y además puede medir la diferencia de temperatura. Con estas funciones de alta gama puede reconocer inmediatamente irregularidades y tomar las medidas pertinentes in situ. El puntero láser integrado le permite adicionalmente situar de forma precisa el campo de medición durante el análisis o registro de imagen. Las imágenes grabadas de la cámara térmica pueden ser transmitidas al ordenador o portátil mediante la tarjeta de memoria SD. También es posible efectuar una medición continua a través del puerto USB de la cámara a un ordenador.

Las imágenes térmicas infrarrojas se analizaron con el programa QUICKREPORT 1.2 SP2 ( PCE Ibérica S.L, Tobarra, España).

\subsection{Análisis estadístico}

Se calculó el promedio y la desviación estándar de la temperatura para cualquier región cutánea de interés seleccionada, se identificó bilateralmente antes y después de correr y fue seleccionada manualmente por el operador, que ignoró las condiciones experimentales y paradigma.

El análisis estadístico se realizó para comparar las diferencias en la distribución de la temperatura cutánea de las zonas seleccionadas antes y después de correr $30 \mathrm{~km}$.

Se utilizó la t student pareada para determinar si las diferencias eran significativas. En el análisis una $\mathrm{P}<0.01$ (con un intervalo del 95\%) se consideró estadísticamente muy significativo. Los datos fueron analizados con el programa informático SPSS, versión 19.0 (SPSS Science, Chicago, Illinois).

\subsection{Consideraciones éticas}

Se siguieron los principios éticos de la Declaración de Helsinki ${ }^{13}$. Los participantes se reclutaron de manera voluntaria, y previo al inicio del estudio, se obtuvo el consentimiento informado de todos los participantes. Se les realizó un consentimiento informado previo a la recogida de datos y a la exploración. En dicho consentimiento se le informaba al participante de los pormenores de la investigación y la explo- ración a la que iban a ser sometidos, así como el proceso de dos días de registro de datos. Los participantes fueron informados de que podían abandonar el estudio en cualquier momento si así lo deseaban. Además también fueron informados del anonimato y carácter confidencial de sus datos.

\section{RESULTADOS}

La Tabla 2 muestra las variaciones termométricas del Miembro Inferior derecho, en ella observamos diferencias significativas en talón, cabeza primer metatarsiano, cabeza segundo metatarsiano, cabeza tercer metatarsiano, cabeza cuarto metatarsiano, cabeza quinto metatarsiano, apófisis estiloides quinto meta, ALI, maléolo interno, maléolo externo, vasto interno, vasto externo, recto femoral, tensor de la fascia lata, inserción cuádriceps, gemelo interno y tendón de Aquiles; variaciones menos significativas en peroneo lateral largo, gemelo externo, semitendinoso, bíceps femoral y semimembranoso y variaciones nada significativas en el tibial anterior.

La Tabla 3 muestra las variaciones termométricas del Miembro Inferior izquierdo, en ella observamos importantes variaciones de temperatura en talón, cabeza primer metatarsiano, cabeza segundo metatarsiano, cabeza tercer metatarsiano, cabeza cuarto metatarsiano, cabeza quinto metatarsiano, apófisis estiloides quinto meta, ALI, maléolo interno, maléolo externo, peroneo lateral largo, vasto interno, vasto externo, recto femoral, tensor de la fascia lata, inserción cuádriceps, gemelo interno, tendón de Aquiles y bíceps femoral; y diferencias poco significativas en tibial anterior, gemelo externo, semimembranoso y semitendinoso.

\section{DISCUSIÓN}

En este trabajo hemos utilizado imágenes de alta resolución térmica de infrarrojos para evaluar los efectos térmicos cutáneos en las extremidades inferiores en la carrera. El estudio experimental consistía en calcular la temperatura de determinadas estructuras anatómicas antes de practicar ningún ejercicio y compararlas con 


\begin{tabular}{|c|c|c|c|c|}
\hline Zona de medida & $\mathbf{N}$ & $\begin{array}{l}\mathrm{T}^{\mathrm{a}} \text { antes de } \\
\text { correr }\end{array}$ & $\begin{array}{l}\mathrm{T}^{\mathrm{a}} \text { después de } \\
\text { correr }\end{array}$ & $\mathbf{P}$ \\
\hline Talón derecho & 32 & $25.39 \pm 1.44$ & $33.47 \pm 1.82$ & 0.001 \\
\hline Cabeza primer meta derecho & 32 & $24.02 \pm 1.32$ & $33.34 \pm 1.66$ & 0.001 \\
\hline Cabeza segundo meta derecho & 32 & $24.16 \pm 1.46$ & $33.52 \pm 1.80$ & 0.001 \\
\hline Cabeza tercer meta derecho & 32 & $23.95 \pm 1.53$ & $33.49 \pm 1.86$ & 0.001 \\
\hline Cabeza cuarto meta derecho & 32 & $23.61 \pm 1.48$ & $33.26 \pm 1.90$ & 0.001 \\
\hline Cabeza quinto meta derecho & 32 & $23.22 \pm 1.28$ & $32.80 \pm 1.86$ & 0.001 \\
\hline Apófisis estiloides quinto meta derecho & 32 & $25.27 \pm 1.26$ & $32.29 \pm 1.71$ & 0.001 \\
\hline ALI derecho & 32 & $27.96 \pm 1.13$ & $34.31 \pm 1.21$ & 0.001 \\
\hline Maleolo interno derecho & 32 & $27.68 \pm 1.00$ & $30.99 \pm 1.26$ & 0.001 \\
\hline Maleolo externo derecho & 32 & $27.69 \pm 0.89$ & $30,94 \pm 1.23$ & 0.001 \\
\hline Peroneo lateral largo derecho & 32 & $30.94 \pm 0.87$ & $31.14 \pm 0.97$ & 0.37 \\
\hline Tibial anterior derecho & 32 & $31.31 \pm 0.88$ & $31.21 \pm 1.54$ & 0.73 \\
\hline Vasto interno derecho & 32 & $30.07 \pm 1.06$ & $31.32 \pm 1.20$ & 0.00 \\
\hline Vasto externo derecho & 32 & $29.65 \pm 1.10$ & $30.28 \pm 1.20$ & 0.03 \\
\hline Recto femoral derecho & 32 & $29.99 \pm 1.28$ & $30.94 \pm 1.34$ & 0.001 \\
\hline Tensor de la fascia lata derecho & 32 & $30.32 \pm 1.39$ & $31.13 \pm 1.32$ & 0.01 \\
\hline Inserción cuádriceps derecho & 32 & $28.71 \pm 1.01$ & $29.64 \pm 1.81$ & 0.02 \\
\hline Gemelo interno derecho & 32 & $31.37 \pm 1.09$ & $31.64 \pm 1.07$ & 0.28 \\
\hline Gemelo externo derecho & 32 & $30.86 \pm 0.95$ & $31.10 \pm 1.25$ & 0.35 \\
\hline Tendón de Aquiles derecho & 32 & $28.36 \pm 0.64$ & $30.10 \pm 1.13$ & 0.001 \\
\hline Semimembranoso derecho & 32 & $30.87 \pm 1.19$ & $31.07 \pm 1.51$ & 0.42 \\
\hline Semitendinoso derecho & 32 & $30.63 \pm 1.20$ & $30.87 \pm 1.47$ & 0.37 \\
\hline Biceps femoral derecho & 32 & $30.68 \pm 1.27$ & $30.96 \pm 1.42$ & 0.32 \\
\hline
\end{tabular}

Tabla 2. Variaciones termométricas del Miembro Inferior derecho. 


\begin{tabular}{|c|c|c|c|c|}
\hline Zona de medida & $\mathbf{N}$ & $\mathrm{T}^{\mathrm{a}}$ antes de correr & $\mathrm{T}^{\mathrm{a}}$ después de correr & $\mathbf{P}$ \\
\hline Talón izquierdo & 32 & $25.31 \pm 1.43$ & $33.37 \pm 1.76$ & 0.001 \\
\hline Cabeza primer meta izquierdo & 32 & $23.77 \pm 1.49$ & $33.13 \pm 1.81$ & 0.001 \\
\hline Cabeza segundo meta izquierdo & 32 & $23.89 \pm 1.63$ & $33.26 \pm 1.89$ & 0.001 \\
\hline Cabeza tercer meta izquierdo & 32 & $23.67 \pm 1.60$ & $33.29 \pm 1.89$ & 0.001 \\
\hline Cabeza cuarto meta izquierdo & 32 & $23.53 \pm 1.46$ & $33.07 \pm 1.81$ & 0.001 \\
\hline Cabeza quinto meta izquierdo & 32 & $23.30 \pm 1.25$ & $32.55 \pm 1.73$ & 0.001 \\
\hline $\begin{array}{l}\text { Apófisis estiloides quinto meta } \\
\text { izquierdo }\end{array}$ & 32 & $25.11 \pm 1.34$ & $31.86 \pm 1.40$ & 0.001 \\
\hline ALI izquierdo & 32 & $27.59 \pm 1.11$ & $34.19 \pm 1.25$ & 0.001 \\
\hline Maleolo interno izquierdo & 32 & $28.11 \pm 0.85$ & $31.4 \pm 0.93$ & 0.001 \\
\hline Maleolo externo izquierdo & 32 & $26.96 \pm 1.19$ & $30.37 \pm 1.40$ & 0.001 \\
\hline Peroneo lateral largo izquierdo & 32 & $30.50 \pm 1.15$ & $30.81 \pm 1.15$ & 0.26 \\
\hline Tibial anterior izquierdo & 32 & $31.06 \pm 0.84$ & $31.26 \pm 1.59$ & 0.47 \\
\hline Vasto interno izquierdo & 32 & $30.02 \pm 1.19$ & $31.27 \pm 1.21$ & 0.001 \\
\hline Vasto externo izquierdo & 32 & $29.41 \pm 1.13$ & $30.09 \pm 1.25$ & 0.02 \\
\hline Recto femoral izquierdo & 32 & $29.87 \pm 1.32$ & $30.84 \pm 1.44$ & 0.001 \\
\hline Tensor de la fascia lata izquierdo & 32 & $30.12 \pm 1.30$ & $30.82 \pm 1.33$ & 0.01 \\
\hline Inserción cuádriceps izquierdo & 32 & $28.64 \pm 1.06$ & $29.62 \pm 1.58$ & 0.01 \\
\hline Gemelo interno izquierdo & 32 & $31.13 \pm 0.87$ & $31.54 \pm 1.07$ & 0.08 \\
\hline Gemelo externo izquierdo & 32 & $30.95 \pm 0.88$ & $31.12 \pm 1.31$ & 0.51 \\
\hline Tendón de Aquiles izquierdo & 32 & $28.30 \pm 0.69$ & $30.03 \pm 1.30$ & 0.001 \\
\hline Semimembranoso izquierdo & 32 & $31.04 \pm 1.20$ & $31.04 \pm 1.65$ & 0.99 \\
\hline Semitendinoso izquierdo & 32 & $30.95 \pm 1.14$ & $30.96 \pm 1.46$ & 0.95 \\
\hline Biceps femoral izquierdo & 32 & $30.69 \pm 1.26$ & $31.06 \pm 1.42$ & 0.20 \\
\hline
\end{tabular}

Tabla 3. Variaciones termométricas del miembro inferior izquierdo. 
las temperaturas obtenidas en esas mismas estructuras anatómicas después de correr $30 \mathrm{~km}$. El mayor trabajo muscular durante la carrera puede provocar una contracción tónica en los músculos de las extremidades inferiores, lo que resulta en sutiles variaciones de la temperatura cutánea.

Ha sido ampliamente demostrado que el ejercicio físico dinámico produce cambios en la temperatura cutánea ${ }^{7,8,14}$ y que el sistema nervioso simpático es el mayor determinante de la temperatura cutánea ${ }^{15}$, no hay conocimiento previo acerca de los efectos térmicos cutáneos asociados con la simple variación de la postura ortostática. Se puede suponer que el aumento en el metabolismo y el calor producido en los músculos involucrados en el control del equilibrio y la postura puede ser transferido a las capas cutáneas ${ }^{11}$.

Las proyecciones cutáneas del Tibial anterior, Gemelo externo, Semimembranoso y Semitendinoso no mostraron variaciones térmicas considerables. En cambio, las variaciones térmicas más importantes fueron las de la zona plantar del pie, Maleolo interno y externo, Peroneo lateral largo, Vasto interno y Vasto externo, Recto femoral, Tensor de la fascia lata, Insercción del cuádriceps, Gemelo interno, Tendón de Aquiles y Bíceps femoral.

Durante la carrera, en la fase de apoyo, el pie está en contacto con el suelo y soporta el peso de todo el cuerpo. El contacto con el suelo se inicia con el borde externo del talón, en este momento el tobillo está en flexión dorsal o extensión, la articulación de la rodilla está extendida y la cadera flexionada, el tibial anterior ejerce una acción estabilizadora del tobillo pero toda la fuerza de la gravedad recae sobre el talón y los maléolos que se encuentran soportando la mayor parte del peso del cuerpo.

El peso se transmite hacia delante, en este momento tenemos la articulación de la rodilla y la cadera en ligera flexión preparadas para la propulsión del peso hacia la parte anterior del pie momento en el que se encuentran la articulación de la rodilla y cadera en extensión preparándose para el despegue del pie y transmitiendo ya el peso al miembro contralateral.
Con esto llegamos a la conclusión que los músculos con menos variaciones térmicas son el tibial anterior que estabiliza el tobillo en la fase de contacto y los músculos que participan en la fase final de propulsión con extensión de cadera y flexión de rodilla, que son los músculos Semimembranoso, Semitendinoso ayudados en la flexión de rodilla por el músculo bíceps femoral.

En este trabajo podemos confirmar que existe una importante variación de temperatura cutánea en determinadas regiones debido al ejercicio realizado durante la carrera, la termometría es un método diagnóstico no invasivo que se puede utilizar en medicina con resultados muy fiables ${ }^{4}$, como no causa ninguna molestia al sujeto se puede utilizar para hacer un seguimiento y observar si el resultado sufre alguna variación al modificar el calzado, el peso del sujeto, o el terreno por ejemplo.

\section{CONCLUSIÓN}

Tras realizar una carrera de $30 \mathrm{~km}$ de distancia los puntos de apoyo del pie que han mostrado una elevación significativa de la temperatura son el talón, arco longitudinal interno, apófisis estiloides del quinto metatarsiano, cabeza primer metatarsiano, cabeza segundo metatarsiano, cabeza tercer metatarsiano, cabeza cuarto metatarsiano y cabeza quinto metatarsiano.

En el Miembro Inferior las zonas que de forma significativa aumentaron la temperatura son talón, cabeza primer metatarsiano, cabeza segundo metatarsiano, cabeza tercer metatarsiano, cabeza cuarto metatarsiano, cabeza quinto metatarsiano, apófisis estiloides quinto metatarsiano, arco longitudinal interno, maléolo interno, maléolo externo, vasto interno, recto femoral, tensor de la fascia lata, insección cuádriceps, tendón de Aquiles y aumentaron la temperatura de forma menos significativa peroneo lateral largo, vasto externo, gemelo interno, gemelo externo y bíceps femoral.

No hubo una variación significativa de la temperatura tras la finalización de la carrera en los músculos semimembranoso, semitendinoso $\mathrm{y}$ tibial anterior. 


\section{BIBLIOGRAFÍA}

1. Diccionario terminológico de ciencias médicas. $13^{\mathrm{a}}$ ed. Barcelona: termometría; p.1208.

2. Ring EF. The historical development of thermometry and thermal imaging in medicine. J Med Eng Technol. 2006; 30(6): 412.

3. Merla A, Romani GL. Functional infrared imaging in medicine: a quantitative diagnostic approach. Eng. Med.Biol.Soc. 2006; 1: 224-231.

4. Bagavathiappan S, Saravanan T, John Philip, Jayakumar T et al. Infrared thermal imaging for detection of peripheral vascular disorders. J Med Phys. 2009; 34(1): 43-47.

5. Mikulska D. Contemporary applications of infrared imaging in medical diagnostics. Ann Acad Stetin. 2006; 52 (1): 35-39.

6. Ring EF, Ammer K. Infrared thermal imaging in medicine. Physol Meas. 2012; 33(3): 33-46.

7. Roback K. An overview of temperature monitoring devices for early detection of diabetic foot disorders. Expert Rev. Med. Devices. 2010; 7(5): 711-719.

8. Ring F. Thermal imaging today and its relevance to diabetes. J. Diabetes Sci Technol. 2010; 4(4): 857862.

9. Zontak A, Sideman S, Verbitsky O, Beyar R. Dynamic thermography: analysis of hand temperature during exercise. Ann. Biomed.Eng. 1998; 26: 988-993.

10. Merla A, Iodice P, Tangherlini A, De Michele G, Di Romualdo S, Saggini R et al. Monitoring skin temperature in trained and untrained subjects throughout thermal video. Eng. Med. Biol.Soc. 2005; 2: 1684-1686.

11. Michele A, Luigi DC, Sandro DR, Silvio I, Antonio F, Gial L et al. Postural adjustment in experimental leg length difference evaluated by means of thermal infrared imaging. Physiol.Meas. 2010; 31: 35-43.

12. Hildebrandt $\mathrm{C}$, Raschner $\mathrm{C}, \mathrm{Ammer} \mathrm{K}$. An overview of recent application of medical infrared thermography in sports in medicine in Austria.Sensors.2010;10(5): 4700-4715.

13. WORLD MEDICAL ASSOCIATION. Declaration of Helsinki: ethical principles for medical research involving human subjects. Available at: http://www.wma.net/en/30publications/10policies/b3/index. html. Accessed january 6, 2012.

14. Ferreira JJ, Mendonça LC, Nunes LA, Andrade Filho AC et al. Exercise-associated thermographic changes in young and lderly subjects. Ann Biomed Eng. 2008; 36: 1420-1427.

15. Gold JE, Cherniack M, Hanlon A, Dennerlein JT et al. Skin temperature in the dorsal hand of office workers and severity of upper extremity musculoskeletal disorders. Int Arch Occup Environ Health. 2009; 82: 1281-1373. 